\title{
Analysis of the restoration of mosque Ali Betchine of Algiers
}

\section{Analyse de la restauration de la mosquée Ali Betchine d'Alger}

\author{
Attari Nassereddine ${ }^{1}$, Mechouari Zineb ${ }^{1}$, Laoues Souad ${ }^{1}$ \\ ${ }^{1}$ Ecole Polytechnique d'Architecture et d'Urbanisme (epau), Alger, Algérie. \\ (Laboratoire Ville Architecture et Patrimoine, LVAP)
}

\begin{abstract}
Ali Betchine is one of the oldest mosques in the city of Algiers of the Ottoman period. Classified as a national monument in 1947. In 1986, UNESCO gave Ali Betchine its universal cultural dimension. This classification of the mosque has resulted in the building currently being protected from any major architectural shuffle in order to protect and preserve it. The Ali Betchine mosque underwent several transformations during the French colonization, it was converted into a church under the name "Notre Dame des Victoires". After the independence of the country, this church was converted into a mosque. The evolution of heritage protection in Algeria can be monitored after a period of follow-up by the country's first regulations on heritage protection. It is noticed that an awareness of the architectural heritage of the country was beginning to emerge. The Ali Betchine mosque underwent restoration work which ended in September 2010 after more than twelve years of closure to the faithful. The paper traces the different stages of its restoration.
\end{abstract}

\begin{abstract}
Résumé : Ali Betchine est l'une des plus anciennes mosquées de la ville d'Alger de la période ottomane. Classée monument historique en 1947 à l'échelle nationale. En 1986, l'UNESCO a donné à Ali Betchine sa dimension culturelle universelle. Ce classement de la mosquée a fait que l'édifice bénéficie actuellement d'une protection qui empêche tout remaniement architectural majeur dans le but de le protéger et le conserver. La mosquée Ali Betchine a subi plusieurs transformations pendant la colonisation française, elle était convertie en église sous l'appellation Notre Dame des Victoires. Après l'indépendance du pays, cette église a été reconvertie en mosquée. On peut suivre l'évolution de la protection du patrimoine en Algérie, et cela après une période de suivi par les premières règlementations propres au pays en matière de protection du patrimoine. On remarque qu'une prise de conscience du patrimoine architecturale du pays commençait à voir le jour. La mosquée Ali Betchine a subi des travaux de restauration qui ont prirent fin en septembre 2010 après plus de douze années de fermeture aux fidèles. Le papier retrace les différentes étapes de sa restauration.
\end{abstract}

\section{Introduction:}

En termes de patrimoine classé, l'Algérie est un pays riche, mais qui se trouve altéré par l'usure $\mathrm{du}$ temps, par les interventions insolites des habitants, les affectations et les modifications de l'occupant français pendant la période coloniale, auquel s'ajoute le manque d'entretien. Face à cet état, des opérations de réhabilitation et de restauration ont étaient entreprises. La restauration est une opération qui a pour but la réinsertion de l'édifice concerné dans son contexte urbain, leur réappropriation en tant que mémoire collective et la restitution de leur architecture originelle dans les limites des possibilités de l'évolution urbaine du contexte physique. En se basant sur des documents fiables, et dans certain cas sur des études de toutes les traces archéologiques selon le cas à restaurer, les restaurateurs doivent respecter le plus proche possible les règles émises par les différentes chartes et conventions internationales.

Nous avons abordé la restauration en tant que mode d'intervention sur le patrimoine bâti, qui par son activation, peut assurer leur conservation et jouer un rôle sur leur durabilité, et l'impact qui va générer sur l'environnement. L'article présente une analyse et une évaluation de la restauration de la mosquée Ali Betchine. 
La mosquée Ali Betchine porte le nom du corsaire vénitien «Piccinini», un grand négociant italien converti à l'islam au mois de radjeb de l'an 1032 de l'hégire (1622) après J-C. Selon Klein, c'était un grand amiral de la flotte algérienne entre 1630 et 1646.

Il est venu volontairement à Alger poursuivre son métier d'armateur de navires destinés à la course qu'il pratiquait déjà dans l'Adriatique. D'autres sources signifient qu'il aurait été d'abord

un captif chrétien, affranchi et converti ensuite à l'islam par Hadj Mustafa BenQara Ali. Différents documents établissent qu'il était le

chef de Ta'ifat al-Riyyas. Ce poste le plaçait au premier rang sur la scène politique, puisqu'il

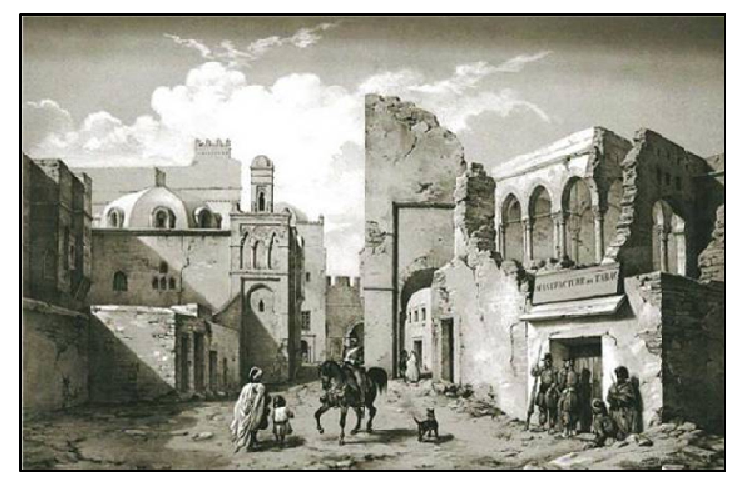

Fig1. la mosquée Ali Betchine, la porte Bab-El-Oued en 1834. Source http://jf.vinaccio.free.fr/site0201/alg02013

Description de la mosquée : La mosquée Ali Betchine possède une grande salle de prière de forme carrée, située à cinq mètres du niveau des rues voisines, repose sur un groupe important de magasins voûtés. On accède, au niveau de la mosquée par deux escaliers donnant; l'un sur la rue Bab-el-Oued, l'autre sur la rue la Casbah.

La salle de prière est surmontée d'une coupole centrale octogonale construite sur quatre piliers épais et des colonnes en pairs (sous chaque arc, il existe deux colonnes, une en tuf et une en pierre), se trouvant dans la cour centrale. Cette cour est entourée de trois galeries couvertes de vingt petites coupoles.

Le minaret qui forme l'angle des rues Bab el Oued et de la Casbah s'élevait autrefois à une hauteur d'environ $26 \mathrm{~m}$; il n'en subsiste au lendemain de la conquête française que la tour de base, qui ne dépasse plus guère $15 \mathrm{~m}$ de haut. $\mathrm{Sa}$ face ouest est encore flanquée de nos jours de la fontaine dite d'Ain- ech-Châra.

Par sa forme et sa construction; le minaret se rattache à un type local des plus simples : une tour carrée qu'aurait surmontée un lanternon carré, qui était considéré comme «gouverneur et capitaine général de la mer et terre ».

Après avoir embrassé la religion musulmane, Piccinini, devenu «Ali Betchine», fait construire cette mosquée sur l'emplacement d'un ancien bagne d'esclaves chrétien sil se fait aider par l'un de ses amis armateur, «Fath Allah Ben Khodja ». 1 Durant la période coloniale, la mosquée Ali Betchine a subi plusieurs modifications et d'autres affectations.

La mosquée a été utilisée comme pharmacie par l'armée française. Puis, servira d'église sous le nom de «Notre Dame des Victoires», de 1843 à 1962. On y aménagea alors une lourde cloche qui ne manqua pas d'endommager le minaret.

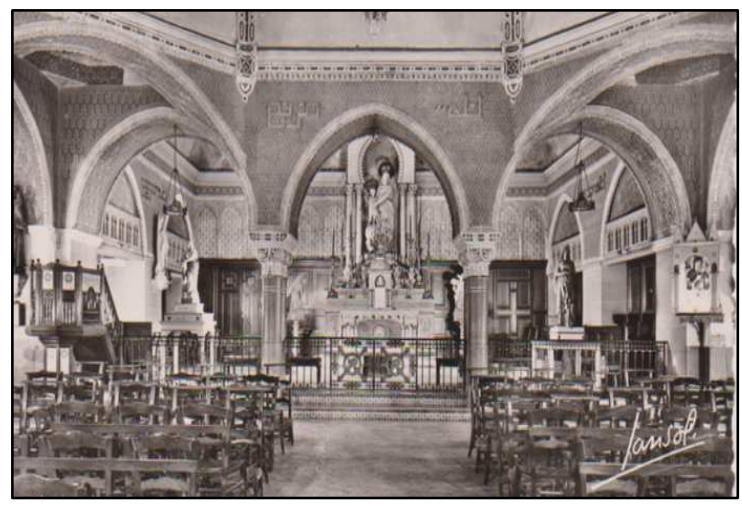

Fig2. L'intérieur de l'église Notre Dames des Victoires (1953)

Source :http://alger-roi.fr/Alger/rue_bab_el_oued

furent défigurés dans leur ensemble lors de leur reconversion coloniale.

Les points d'appui, entre la coupole et les galeries, sont constitués par les piliers cruciformes, situés aux quatre angles de la salle de prière. Des coupolettes, à base octogonale épaulent la coupole centrale et complètent la couverture du carré central et en occupant les quatre angles de la calotte octogonale. Entre les piliers d'angles et sur chaque face de la salle carrée, se trouvent quatre colonnes en ciment groupées par deux. Les piliers et les colonnes reçoivent des arcs brisés, situés au-dessus de la grande coupole. Les arcs séparent la salle de prière des galeries qui l'entourent sur ces quatre côtés. Les galeries sont couvertes de coupolettes, à la base octogonale, reliées au carré de base par des pendentifs, et qui sont séparées par des doubleaux. On note simplement que les piliers d'angles sont remplacés par seize colonnes, groupés par quatre à chaque angle. D'autre part, les galeries qui bordent le carré central ne sont pas couvertes totalement par des coupolettes. Celles-ci n'apparaissent qu'aux angles des galeries; et en avant du mihrab, le reste est couvert par des voûtes d'arêtes. Des berceaux surbaissés remplacent les coupolettes et complètent la couverture du carré central. 


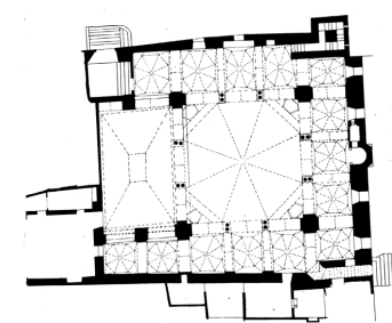

Fig 3. Plan de la mosquée Ali Betchine [2)

Cet intérieur était blanchi à la chaux et n'offrait aucune décoration. A droite, en entrant par la rue de la Casbah, en face du mihrab et sur le dépourvu de galerie, se trouvait une cour à ciel ouvert renfermant quelques arbres et un jeu d'eau. C'est dans cette partie, qu'ont été installés le chœur et la sacristie de l'église N. D. des Victoires.

Si le minaret n'é été que partiellement dérasé en 1860, les latrines et les lieux d'ablutions ont été en revanche entièrement démolis. Leur emplacement a été englobé, selon Devoulx, dans l'habitation portant le $\mathrm{n}^{\circ} 2$ de la rue de la Casbah.

La façade septentrionale, donnant sur la rue de la Casbah, et percée de trois fenêtres ogivales, contient au rez-de-chaussée nef boutiques dont sept seulement sont apparentes aujourd'hui, et la grande porte d'entrée, que quatre marches assez larges relient à la nef. Cette issue est actuellement clôturée par une porte sculptée, d'un assez joli travail, qui appartenait primitivement à la mosquée Ketchaoua, et qui a été placée là par les Français eu 1843. Audessus de la rosace médiale de chacun des deux battants de cette porte, on lit la phrase suivante, qui se détache en relief comme le reste de l'ornementation: 《ماشاءالله 》, que la volonté de Dieu s'accomplisse. On attribue ces sculptures au Maître «Ahmed ben Lablabtchi » le responsable de la corporation des menuisiers. (3)

Sur le plan architectural, l'édifice religieux est considéré parmi les premières et authentiques typologies de mosquées ottomanes actuellement subsistantes. Sa superficie s'étend sur environ $500 \mathrm{~m}^{2}$.

\section{Les travaux de restauration de la mosquée Ali Betchine :}

\section{a. Consolidation :}

Les fissures présentes sur les murs, les arcs et les coupoles de la mosquée ont été réparées selon deux procédés différents en fonction de leur degré d'importance :

- Les fissures ayant une ouverture inférieure à $3 \mathrm{~mm}$ de largeur ont été réparées avec des agrafes métalliques associées avec des injections sous pression,

- Les fissures ayant une ouverture supérieure à $3 \mathrm{~mm}$ de largeur ont été réparées avec du

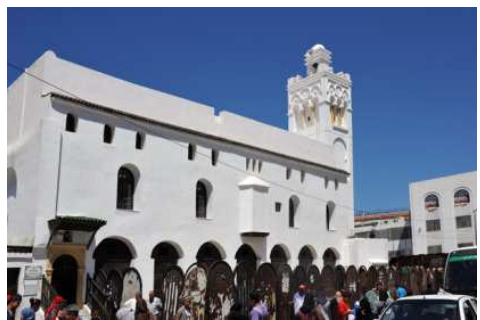

Fig 5. Photo de la mosquée 2016

mortier armé en treillis soudés avec injection sous pression.

Pour cela, il faudra noter qu'avant tout, ils ont décapé les enduits intérieurs et extérieurs pour permettre des mesures précises de l'ouverture des fissures.

La réparation avec les agrafes métalliques a été exécutée de la manière suivante :

1- Après le décapage des enduits, ils ont procédé à des percements dans la maçonnerie de $30 \mathrm{~cm}$ de part et d'autre de la fissure, à une profondeur d'au moins $20 \mathrm{~cm}$, et un espacement de $15 \mathrm{~cm}$ dans le sens de la longueur de la fissure. Les trous avaient un diamètre de $25 \mathrm{~mm}$.

2- Ensuite, ils ont mis en place des agrafes en acier maxydable de diamètre 8 et $1.00 \mathrm{~m}$ de longueur, dont les parties encastrées ont été enrobées de mortier bâtard.

3- Les fissures ont été ensuite colmatées par injection sous pression d'un lait de chaux et ciment avec des proportions équivalentes (01 volume de chaux, 01 volume de ciment 250), et l'injection a été faite après le nettoyage des fissures à l'eau.

La chaux était de type hydraulique, la chaux aérienne est formellement proscrite pour les injections.

La réparation des fissures avec le mortier armé a été exécutée comme suit :

1- Apres décapage de l'enduit et piquage des joints à $1 \mathrm{~cm}$ de profondeur, il été procédé à la mise en place de l'armature en treillis soudés de $\varnothing 4$ ou $\varnothing 5$ qui doit déborder de $1 \mathrm{~m}$ de chaque côté de la fissure et maintenue à $0.5 \mathrm{~cm}$ de la surface de la maçonnerie.

2- L'armature en treillis soudés a été accrochée à son appui à l'aide de tiges métalliques de $8 \varnothing$ en acier inoxydable, disposées chaque $50 \mathrm{~cm}$ et encastrées dans toute l'épaisseur de la maçonnerie. Elles été enrobées de mortier bâtard dans des trous de $25 \mathrm{~mm}$ de diamètre.

3- Ils ont exécuté ensuite l'enduit en trois couches (ou deux couches pour l'extérieur), dont la composition et le dosage étaient précis. 
4- Les fissures ont été colmatées par injection sous pression d'un mortier bâtard après avoir été nettoyées à l'eau. Le dosage du mortier pour l'injection est comme suit :

$\checkmark$ Ciment : 01 volume,

$\checkmark$ Chaux hydraulique : 01 volume,

$\checkmark$ Sable : 07 volumes.

5- Des chainages horizontaux ont été exécutés au-dessus des murs et arcs de la salle de prière. Ils ont des dimensions de 90 x $20 \mathrm{~cm}$ (ferraillés en $8 \mathrm{~T} 12$, cadre $\varnothing 6$ chaque $20 \mathrm{~cm}$ ), et $30 \times 20 \mathrm{~cm}$ (ferraillés en 4 T12, cadre $\emptyset 6$ chaque $20 \mathrm{~cm}$ ).

Les coupolettes restituées reposeront sur des chainages horizontaux de dimensions $20 \mathrm{x}$

\section{b. Intervention dans les locaux- voûtes :}

Un décapage a été nécessaire pour réaliser la nature et l'état réel des murs et voûtes, où plusieurs interventions ont été déjà remarquées. Une attention particulière a été portée aux voûtes, celles-ci étaient mal aérées avec la présence du phénomène de condensation qui humidifie les murs et par conséquent, rend le crépi friable. La présence d'un puits au sein même de l'épaisseur d'un mur porteur laisse à considérer les remontées capillaires susceptibles d'altérer la base des murs.

\subsection{1. e. Planchers :}

- Au-dessus du voligeage, ils ont procédé au coulage d'une chape en béton de $5 \mathrm{~cm}$ d'épaisseur armée en treillis soudés avec interposition d'un film polyane.
20 (ferraillés en 4 T12, cadre $\emptyset 6$ chaque $20 \mathrm{~cm})$.

Des chainages verticaux et horizontaux ont été exécutés dans les locaux. Ils ont des dimensions de $20 \times 20 \mathrm{~cm}$ et étaient ferraillés en $4 \mathrm{~T} 12$, cadres $\varnothing 6$ chaque $20 \mathrm{~cm}$.

Des étaiements traditionnels sont effectués pour la reconstruction de planchers d'autres étaiements de type chevalement sont fait pour soutenir les naissances d'arcs dans le but est de reconstituer les chapiteaux endommagés. Des mesures à l'ultrason aux scléromètres tendres et sures pour déterminer la résistance des colonnes composées des tuf et de pierre.

- Ils ont procédé à l'exécution de planchers collaborant en poutre de bois (solives) et chape armée en treillis soudés dans la salle de prière et les locaux et au-dessus de l'accès à la terrasse à partir du minaret.

- Les poutres ont des dimensions de $7 \mathrm{x}$ $14 \mathrm{~cm}$ posées chaque $20 \mathrm{~cm}$ entraxes. Elles sont encastrées dans les chainages horizontaux du plancher.

- Au-dessus des poutres, un voligeage est posé, composé de planches en bois de $2 \mathrm{~cm}$ d'épaisseur.

- Les éléments en bois ont reçu au préalable un traitement avec un produit hydrofuge

\section{La salle de prière après la restauration :}

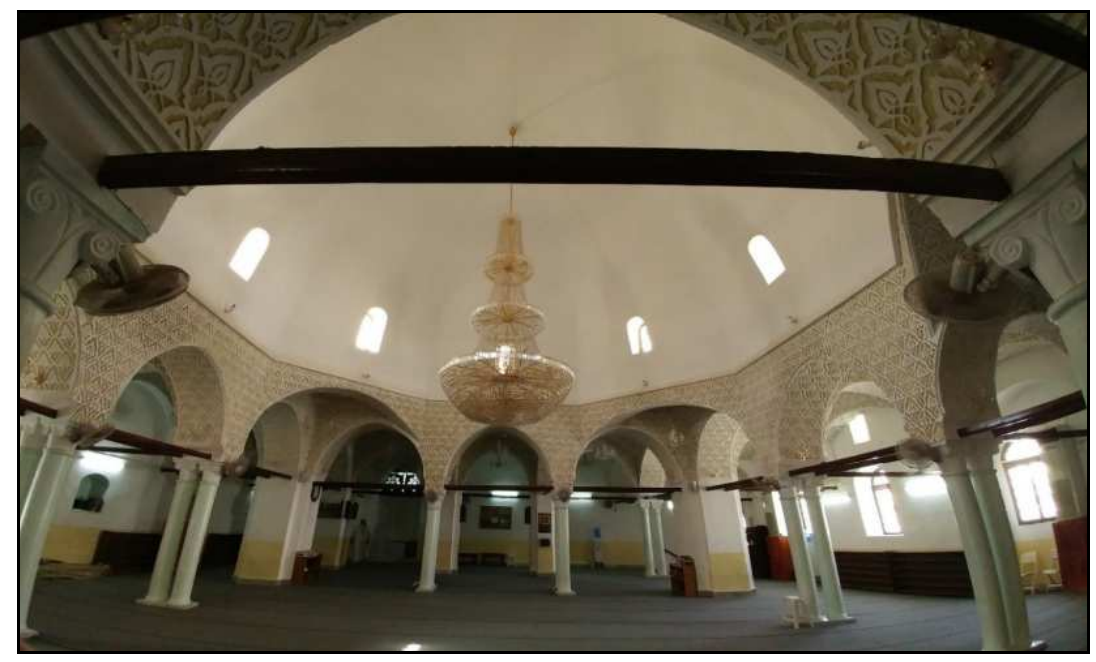

Fig 6. Vue sur l'ensemble de la salle de prière, 15/09/2016. Source : Auteur

\section{Conclusion :}

La restauration d'un patrimoine bâti ancien, à l'exemple de notre cas d'étude la mosquée Ali 
Betchine, et cela en vue, de garantir sa conservation dans de bonnes conditions, n'est pas une mince affaire, il s'agit surtout, d'une opération compliquée, délicate et exigeante. La décision de restaurer un édifice suppose une volonté de prise en charge à long terme. La restauration n'est que le premier pas d'un processus de conservation et de mise en valeur qui ne devra jamais cesser.

\section{Référence :}

1. Chergui Samia, 2011, Les mosquées d'Alger; construire, gérer et conserver (XVI-XIX siècles), 1ere éd., P U De Paris-Sorbonne, Paris, 371p .

2. Dokali Rachid, 1974, Les Mosquées de la période turque à Alger, 1ere éd, 1ere éd., SNED, Alger, 127p.

3. Kerdoune Aicha, 2011, Les Mosquées historiques de la ville d'Alger, 1ere éd., Alpha Editions, Telmcen, $256 \mathrm{p}$.

4. Duval George, 1990, Restauration et réutilisation des monuments anciens, techniques contemporaines, 1ere éd., Mardage, Belgiun, 1990, 290p. 